\title{
Alternative Surgical Strategy for AxiaLIF Pseudarthrosis: A Series of Three Case Reports
}

\author{
Jan K. G. Louwerens ${ }^{1}$ Diederik Groot ${ }^{2}$ Dennis C. van Duijvenbode ${ }^{1}$ Maarten Spruit $^{3}$
}

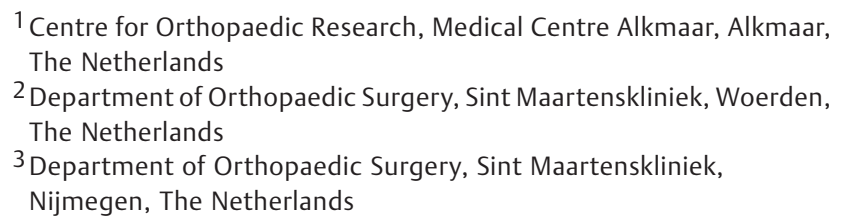

Address for correspondence Diederik Groot, MD, Department of Orthopaedic Surgery, Sint Maartenskliniek, Polanerbaan 2, 3447 GN, Woerden, The Netherlands (e-mail: d.groot@maartenskliniek.nl).

Evid Based Spine Care J 2013;4:143-148.

\begin{abstract}
Keywords

- lumbar spine

- interbody fusion

- axial

- presacral

- pseudarthrosis

- revision surgery

- AxiaLIF

Study Design Retrospective case series.

Objective The objective of this study is to describe an alternative technique to attain interbody lumbar fusion in the event of pseudarthrosis after axial lumbar interbody fusion (AxiaLIF) and to assess its safety.

Methods Three patients who suffered from pseudarthrosis after AxiaLIF underwent revision surgery with a DEVEX cage (DePuy Synthes, Raynham, MA, United States) through an anterior approach. We report technical details as well as clinical and radiological results at 12 months follow-up.

Results Preoperative symptoms resolved in all cases. There were no perioperative complications. One patient had a deep venous thrombosis at postoperative day 9. A decrease in visual analog scale score for pain was observed, from 8.67 preoperatively to 2 postoperatively at final follow-up. Radiographic workup after 12 months showed no sign of implant failure or loosening, and fusion was obtained in all cases.

Conclusion Anterior fusion with a DEVEX cage in front of a TranS1 screw (TranS1 screw, Inc., Wilmington, North Carolina, United States) for AxiaLIF pseudarthrosis is safe and effective.
\end{abstract}

\section{Introduction}

The axial lumbar interbody fusion (AxiaLIF) technique is a minimally invasive percutaneous technique to attain fusion at L5-S1 and L4-S1. ${ }^{1,2}$ The technique includes a small paracoccygeal incision that allows percutaneous access to the presacral "safe zone" area with use of sequential dilation followed by discectomy and placement of a threaded rod (TranS1 screw, Inc., Wilmington, North Carolina, United States). ${ }^{2,3}$ AxiaLIF has the potential to reduce the rate of associated surgical complications, such as vascular injury and sympathetic disruption, by avoiding dissection and disruption of functional tissue surrounding the surgical site. ${ }^{4}$ These advantages are replaced by a significant chance of rectal and bowel injury. ${ }^{5}$ There is limited literature available on long-term follow-up, but radiographic fusion rates of $94 \%$ at 2 years have been reported. ${ }^{6}$

Indications for AxiaLIF are the same for traditional fusion procedures, ${ }^{5}$ such as anterior lumbar interbody fusion, ${ }^{7}$ transforaminal lumbar interbody fusion (TLIF), ${ }^{8}$ and posterior lumbar interbody fusion (PLIF) ${ }^{9}$ : low back pain associated with grade I or II spondylolisthesis, degenerative disc disease, postlaminectomy instability, or pseudarthrosis at L4-L5 of L5-S1.

Complications of spinal fusion surgery using the AxiaLIF include the following: superficial infections, pseudarthrosis, and failure of osteosynthesis material in up to $23.5 \%{ }^{10,11}$ received

March 17, 2013

accepted

July 18, 2013 (c) 2013 Georg Thieme Verlag KG Stuttgart · New York
DOI http://dx.doi.org/ 10.1055/s-0033-1357357. ISSN 1663-7976. 
Revision percentages for pseudarthrosis of $8.8 \%$ at 3-year follow-up have been reported. ${ }^{5}$

In this technical note, we present three cases of pseudarthrosis after using the AxiaLIF technique. There is only limited literature on revision techniques in the case of pseudarthrosis after the AxiaLIF procedure. Both anterior and posterior revision strategies can be used to achieve lumbar interbody fixation, with or without removing the AxiaLIF rod. ${ }^{11,12}$ One case report describes a minimally invasive revision of the AxiaLIF rod through the same presacral approach, replacing it with a larger diameter rod. ${ }^{13}$

The purpose of this case report is to describe an alternative to attain fusion in the event of pseudarthrosis through an anterior retroperitoneal approach and the use of an intervertebral cage in front of the TranS1 screw.

\section{Method and Report of Three Cases}

Pseudarthrosis after AxiaLIF is illustrated with three case presentations. All patients had progressive postoperative back pain and were not responsive to conservative treatment. The operations were performed in the same clinic using the surgical method described below.

The first case is of a 37-year-old woman who presented with severe back pain and sacral pain after AxiaLIF level L5-S1 with additional transfacet screws performed for symptomatic monosegmental degenerative disc disease refractory to years of conservative care. Preoperative computed tomographic (CT) scan showed that the TranS1 screw and transfacet screws were intact. However, there was no sign of bridging intervertebral bone and there was minimal radiolucency around the AxiaLIF rod (-Figs. 1 and 2). An anterior revision arthrodesis level L5-S1 with placement of a DEVEX cage (DePuySynthes) was performed 10 months after the initial AxiaLIF because of persistent back pain. Radiography, at 3 months postoperatively, showed a stable situation with the DEVEX cage anterior of the TranS1 screw (-Figs. 3 and $\mathbf{4}$ ).

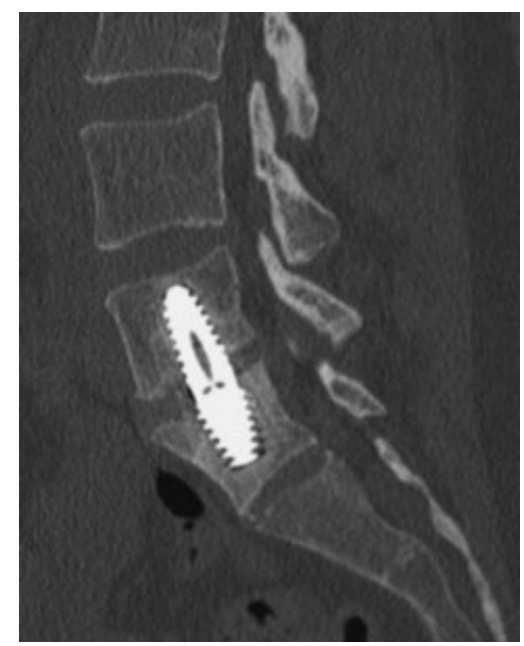

Fig. 1 Case 1. Preoperative computed tomography showing radiolucency around the AxiaLIF rod.

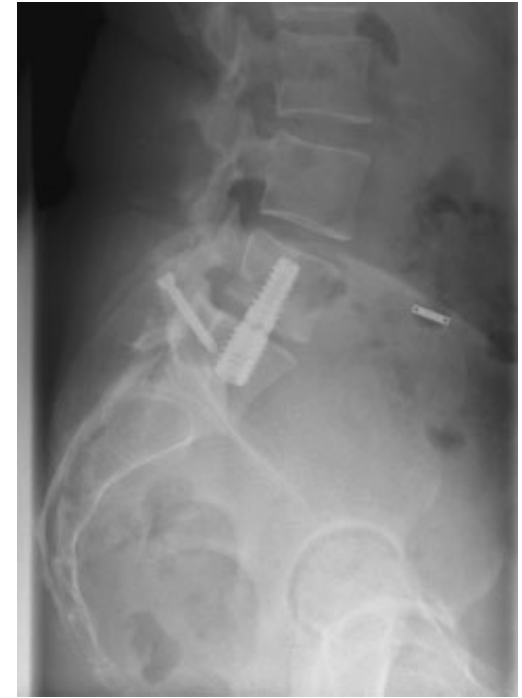

Fig. 2 Case 1. Preoperative lateral X-ray of lower lumbar spine (X-LWK) showing no signs of intervertebral bone formation.

The second case is a 28-year-old man who presented with severe lower back pain and radiculopathy in the right leg after AxiaLIF level L5-S1 with posterior pedicle screw fixation performed for symptomatic grade 1 isthmic spondylolisthesis refractory to 6 months of conservative care (-Figs. 5 and $\mathbf{6}$ ). Anterior revision arthrodesis level L5-S1 with placement of a DEVEX cage in combination with revision of the broken pedicle construct was performed 9 months after AxiaLIF. Radiography, 3 months postoperative, showed a solid construct at level L5-S1 with the DEVEX cage in front of the TranS1 screw and the broken pedicle screws still in situ (-Figs. 7 and 8 ).

The third case is of a 36-year-old woman who presented with severe lower back pain after AxiaLIF level L5-S1 with additional disc prosthesis (Cadisc, Ranier Technology, Cambridge, United

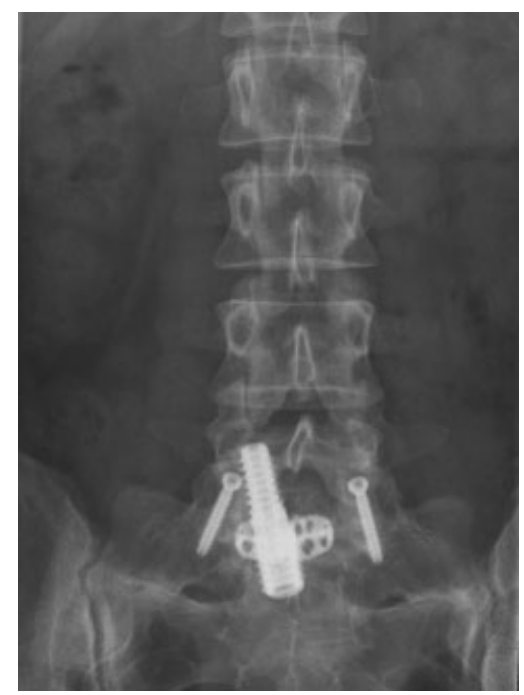

Fig. 3 Case 1. Three months postoperative anteroposterior and lateral X-LWK. 


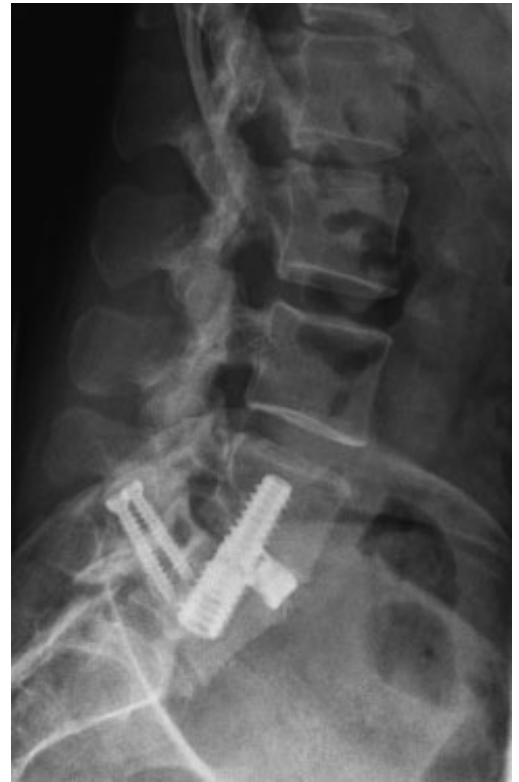

Fig. 4 Case 1. Three months postoperative anteroposterior and lateral X-LWK.

Kingdom) L4-L5 performed for symptomatic degenerative disc disease refractory to more than 10 years of conservative care. The disc prosthesis was placed 1 year after the initial AxiaLIF. Preoperative CT showed a wide intervertebral disc space level L4-L5 with intervertebral disc prosthesis and TranS1 screw L5S1. There was no sign of bridging intervertebral bone at level L5S1, consistent with pseudarthrosis (- Figs. 9 and 10). An anterior revision level L5-S1 arthrodesis was performed with removal of the intervertebral disc prosthesis and placement of a syncage

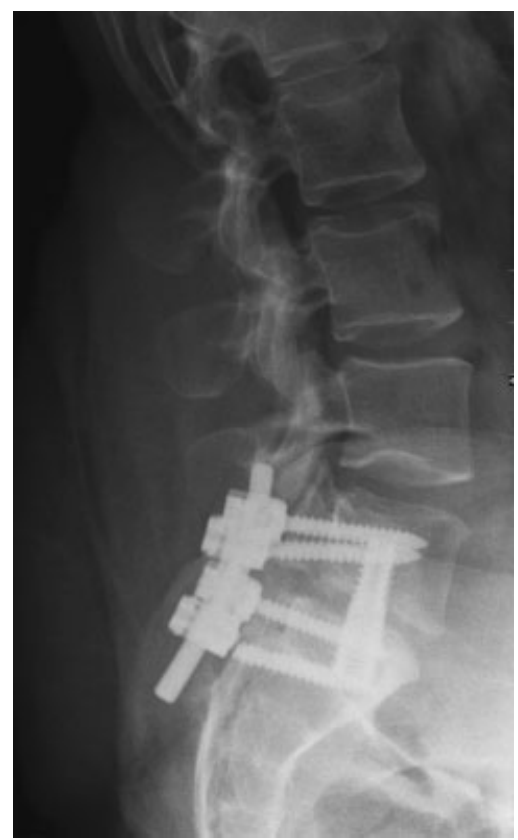

Fig. 5 Case 2. Preoperative computed tomography showing radiolucency around the AxiaLIF rod.

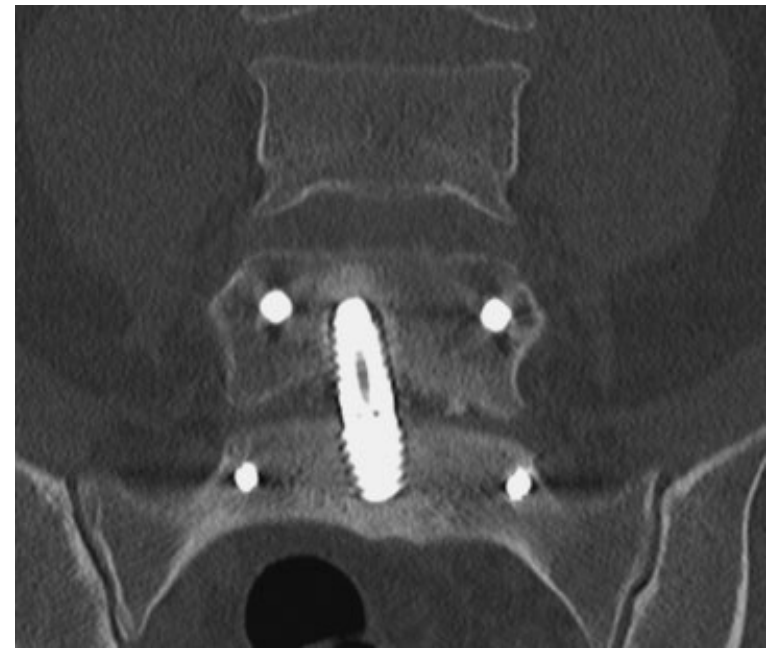

Fig. 6 Case 2. Preoperative lateral X-LWK showing the broken pedicle screw at level S1.

with autologe bonegraft followed by placement of a DEVEX cage anterior of the TranS1 screw. The construct was reinforced with posterior pedicle screws level L4-S1 until full consolidation was achieved. Revision surgery was performed 4 years after the initial AxiaLIF. Radiography, at 6 months postoperative, showed a solid arthrodesis level L4-S1 with the osteosynthesis material in place (-Figs. 11 and 12).

Standard anteroposterior and lateral radiographs in combination with sagittal and coronal reconstruction by CT were used to assess fusion. Recent literature recommend these imaging modalities to be used in the assessment of pseudarthrosis. ${ }^{14}$ The TranS1 screw was left in situ as there was neither preoperative suspicion of excessive bone resorption around the device nor were there signs of infection. Clinical

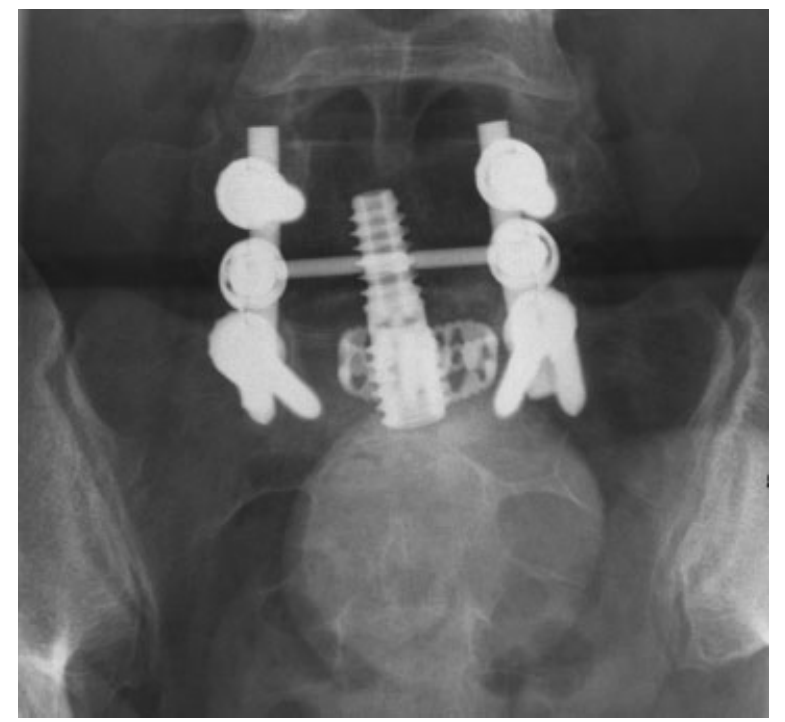

Fig. 7 Case 2. Three months postoperative anteroposterior and lateral X-LWK. 


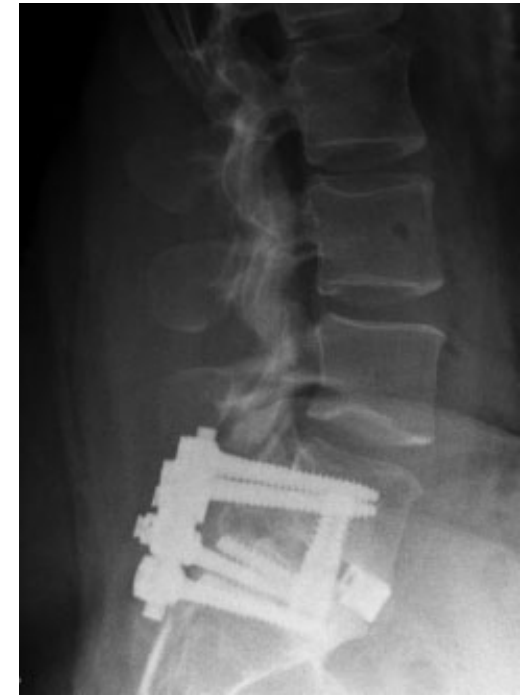

Fig. 8 Case 2. Three months postoperative anteroposterior and lateral X-LWK.

and radiological examinations were performed at 3, 6, and 12 months after surgery.

\section{Surgical Technique}

A median retroperitoneal approach was performed to access the L5-S1 disc space. The anterior longitudinal ligament was excised, and in all cases, there was residual disc tissue present. There was no sign of intercorporal bone formation. A discectomy L5-S1 was performed around the AxiaLIF rod. Next, curved curettes were used to remove osseous overgrowth, widen the aperture, and rasp until bleeding, cancellous bone was encountered. Allograft bone was placed around the AxiaLIF rod. A DEVEX cage, commonly used for TLIF, was filled with the same bone graft and placed anterior of the

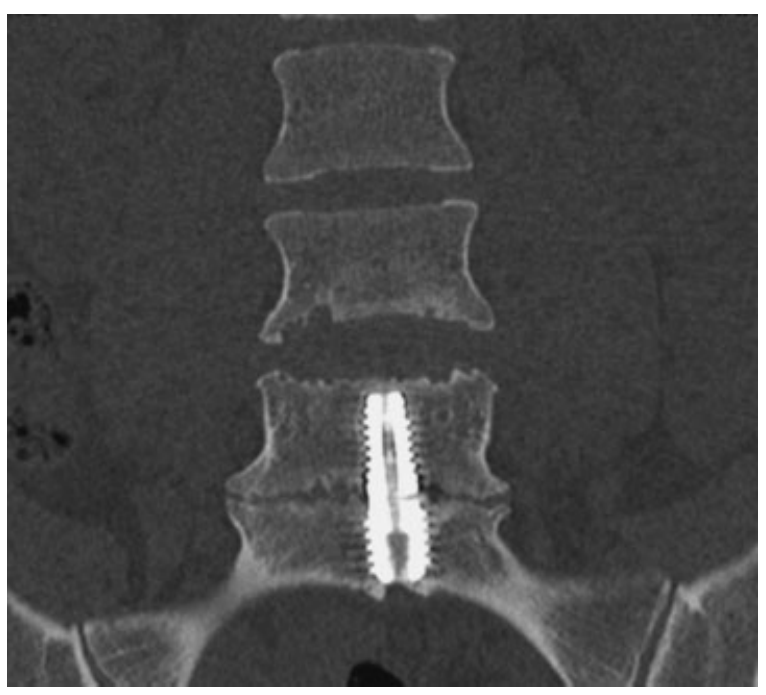

Fig. 9 Case 3. Preoperative computed tomography showing a wide intervertebral disc space level L4-L5 with intervertebral disc prosthesis and no sign of intervertebral bone formation.

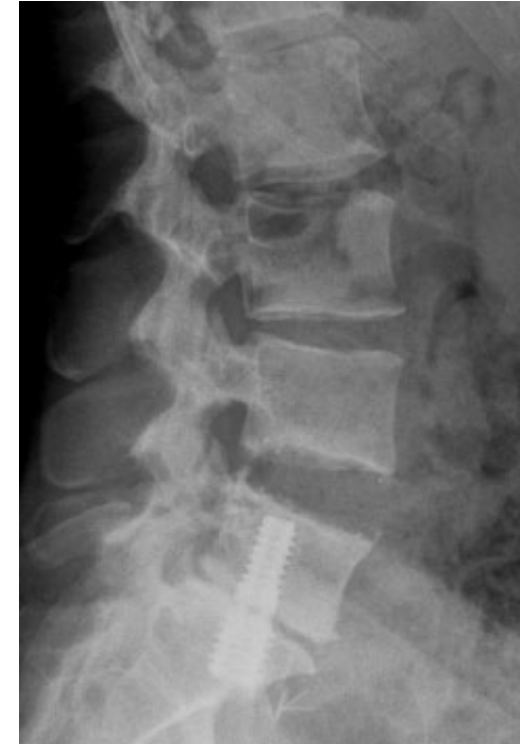

Fig. 10 Case 3. Preoperative lateral X-LWK showing no signs of intervertebral bone formation.

implant with firm impaction. Additional bone graft was placed in front of the cage in the remaining disc space. Fixation with an additional anterior tension band plate was not necessary. The surgical site was closed according to standard methods.

\section{Results}

There were no perioperative local or general complications. The mean operative blood loss was $125 \mathrm{~mL}$. The postoperative course of case 3 was complicated by a deep vein thrombosis in the left leg on day 8 postoperatively, which was treated according to protocol. All patients were mobilized the first day after surgery without lumbar support. Postoperative

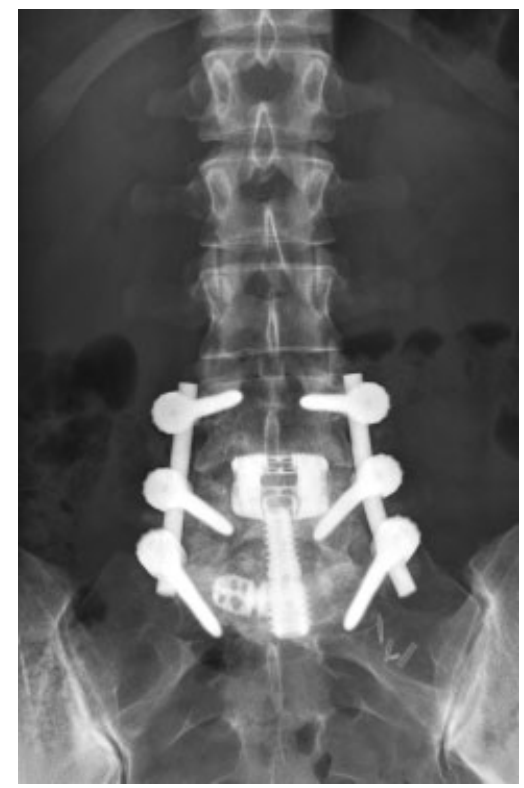

Fig. 11 Case 3. Six months postoperative anteroposterior and lateral X-LWK. 


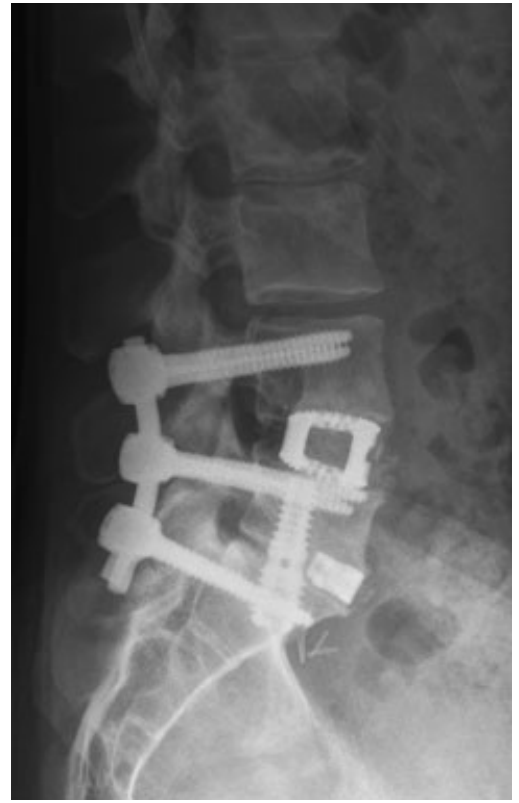

Fig. 12 Case 3. Six months postoperative anteroposterior and lateral X-LWK.

radiographs showed good position of the cage in front of the Trans1 screw. All three patients were relieved of their preoperative symptoms. A decrease in visual analog scale score for pain was observed from a mean score of 8.67 preoperatively to 2 postoperatively at final follow-up. At 12 months followup, the patients reported to experience no more pain or limitations in daily activities. Radiographic workup after 12 months showed no sign of implant failure or loosening, and fusion was obtained in all cases. We did not do CT scans to confirm bony fusion.

\section{Discussion}

The minimal invasive AxiaLIF approach has the potential to reduce the rate of complications by avoiding dissection and disruption of functional tissue surrounding the surgical site. But as with all techniques that share the goal of fusion, the ultimate goal of treatment obviously is to obtain fusion and to avoid pseudarthrosis. Although biomechanical studies reveal that the intact annulus contributes to AxiaLIF stability ${ }^{15}$ and although the approach results in minimal iatrogenic instability, the pitfalls of this technique are incomplete disc removal and insufficient end plate preparation. It remains a challenge to add bone into the intervertebral disc space and hence to obtain bony fusion. Therefore, pseudarthrosis is a risk, and revision surgery for pseudarthrosis is reported in $8.8 \%$ after 3 years follow-up. ${ }^{5}$

Revision surgery after AxiaLIF in the setting of pseudarthrosis is a challenge with the TranS1 in situ. Removal of the implant may be unavoidable in cases of infection or extensive bone loss. Literature reports two options for implant removal: either by an anterior sacral resection ${ }^{12}$ or by a percutaneous retrieval of the device using the same presacral working corridor as for implantation. ${ }^{16}$ For surgeons unfamiliar with the presacral approach, a revision through this corridor is not appealing.

Alternatives for revision strategy with the implant in situ have been reported. A relatively easy approach is posterolateral instrumented fusion. ${ }^{11}$ This could be combined with an additional PLIF. PLIF cages may be introduced on either side of the TranS1 screw to achieve intervertebral fusion. ${ }^{11}$ A different approach is to go anterior and aim for anterior fusion while leaving the TranS1 in situ. As anterior cage footprints will not fit, a smaller footprint cage in front of the TranS1 will add to stability of the construct and promote fusion. We consider this technique to be safe and simple. The key to success is full anterior disc exposure that allows complete discectomy. The anterior retroperitoneal approach is attractive to achieve this. The TLIF-cage used in all of our patients fits perfectly anterior of the TranS1 screw because of its shape, and in combination with extra bone graft for fusion, it provides the necessary stability to obtain anterior intervertebral fusion. We consider our technique to be easier compared with placing PLIF cages on either side of the TranS1 screw or by re-entering the presacral zone that might not be a "safe zone" to surgeons unfamiliar with that approach.

\section{Conflict of Interest \\ None}

\section{Funding}

There was no funding received for this work.

\section{References}

1 Carl A, Ledet E, Oliveira C, et al. Percutaneous axial lumbar spine surgery. In: Perez-Cruet MJ, Khoo LT, Fessler RG, eds. An Anatomic Approach to Minimally Invasive Spine Surgery. St Louis, MO: Quality Medical Publishing; 2006:654-670

2 Cragg A, Carl A, Casteneda F, et al. New percutaneous access method for minimally invasive anterior lumbosacral surgery. J Spinal Disord Tech 2004;17:21-28

3 Marotta N, Cosar M, Pimenta L, et al. A novel minimally invasive presacral approach and instrumentation technique for anterior L5-S1 intervertebral discectomy and fusion: technical description and case presentations. Neurosurg Focus 2006;20(1):E9

4 Schwender JD, Holly LT, Rouben DP, et al. Minimally invasive transforaminal lumbar interbody fusion (TLIF): technical feasibility and initial results. J Spinal Disord Tech 2005;18(Suppl):S1-S6

5 Lindley EM, McCullough MA, Burger EL, et al. Complications of axial lumbar interbody fusion. J Neurosurg Spine 2011;15(3): 273-279

6 Tobler WD, Gerszten PC, Bradley WD, et al. Minimally Invasive Axial Presacral L5-S1 Interbody Fusion: two-year clinical and radiographic outcomes. Spine 2011;36:1296-1301

7 Burkus JK, Gornet MF, Dickman CA, et al. Anterior lumbar interbody fusion using rhBMP-2 with tapered interbody cages. J Spinal Disord Tech 2002;15:337-349

8 Freeman BJ, Licina P, Mehdian SH. Posterior lumbar interbody fusion combined with instrumented postero-lateral fusion: 5-year results in 60 patients. Eur Spine J 2000;9:42-46 
Louwerens et al.

9 Rosenberg WS, Mummaneni PV. Transforaminal lumbar interbody fusion: technique, complications, and early results. Neurosurgery 2001;48(3):569-574

10 Tobler WD, Ferrara LA. The presacral retroperitoneal approach for axial lumbar interbody fusion: a prospective study of clinical outcomes, complications and fusion rates at a follow-up of two years in 26 patients. J Bone Joint Surg Br 2011;93(7):955-960

11 Hofstetter CP, James AR, Hartl R. Revision strategies for AxiaLIF. Neurosurg Focus 2011;31(4):E17

12 DeVine JG, Gloystein D, Singh N. A novel alternative for removal of the AxiaLif (TranS1) in the setting of pseudarthrosis of L5-S1. Spine J 2009;9(11):910-915

\section{Commentary}

\author{
Daryl R. Fourney ${ }^{1}$ \\ ${ }^{1}$ Division of Neurosurgery, University of Saskatchewan, Saskatoon, \\ Canada
}

Louwerens et al describe a surgical treatment strategy for dealing with pseudoarthrosis after axial lumbar interbody fusion. The main problem is providing enough bone in the intervertebral space to obtain fusion with the TranS1 screw (TranS1 screw, Inc., Wilmington, North Carolina, United States) in situ. Removal of the screw has been advocated by both anterior (presacral resection) ${ }^{1}$ and posterior ${ }^{2}$ approaches, although both are technically challenging.

Louwerens et al have shown excellent short-term results with simply placing an interbody cage anterior to the TranS1 with supplemental posterior stabilization. A major limitation of this report was that although radiographs were apparently followed to 12 months (see Results section), the figures provided were at only 3 months (cases 1 and 2) and 6 months (case 3 ). In addition to longer follow-up, computed tomography would be helpful to confirm bony fusion.

\section{Editorial Perspective}

The technical discussion of how to treat complications associated with more recently developed minimally or less invasive procedures again raises the question of the efficiency of these implants after release into the general public. Release of many of these "newer" fusion techniques is frequently based upon underpowered studies performed by dedicated "experts" with many flaws in study-design validity. True reoper-
13 Cohen A, Miller LE, BlockJE. Minimally invasive presacral approach for revision of an Axial Lumbar Interbody Fusion rod due to fall related lumbosacral instability: a case report. J Med Case Reports 2011;5:488

14 Goldstein C, Drew B. When is a spine fused? Injury. Int. J. Care Injured 2011;42:306-313

15 Ledet E, Tymeson M, Salerno S, et al. Biomechanical evaluation of a novel lumbosacral axial fixation device. J Biomech Eng 2005; 127:929-933

16 Aryan HE, Newman CB, Gold JJ, et al. Percutaneous axial lumbar interbody fusion (AxiaLIF) of the L5-S1 segment: initial clinical and radiographic experience. Minim Invasive Neurosurg 2008; 51(4):225-230
I agree that removal of the TranS1 may not be necessary unless there is major complication such as infection or neurological compromise. If the TranS1 had been placed too ventrally across the disc space to allow a cage in front of it, then posterior lumbar interbody cages may be placed on either side of it. However, the simplest thing to do is a posterolateral instrumented fusion.

\section{References}

1 DeVine JG, Gloystein D, Singh N. A novel alternative for removal of the AxiaLif (TranS1) in the setting of pseudarthrosis of L5-S1. Spine J 2009;9(11):910-915

2 Aryan HE, Newman CB, Gold JJ, Acosta FL Jr, Coover C, Ames CP. Percutaneous axial lumbar interbody fusion (AxiaLIF) of the L5-S1 segment: initial clinical and radiographic experience. Minim Invasive Neurosurg 2008;51(4):225-230 ation rates in a low back pain fusion population are likely underreported and the complexity of reoperations becomes increasingly daunting, such as in this case. It is always helpful to humbly remember that the term "technically challenging" is all but a euphemism for "increased likelihood of a serious complication." 